\title{
Article
}

\section{Large-Scale Model of the Milky Way: Stellar Kinematics and the Microlensing Event Timescale Distribution in the Galactic Bulge}

\author{
Bissantz, Nicolai, Debattista, Victor P and Gerhard, Ortwin
}

Available at https://clok.uclan.ac.uk/17008/

Bissantz, Nicolai, Debattista, Victor P orcid iconORCID: 0000-0001-7902-0116 and Gerhard, Ortwin (2004) Large-Scale Model of the Milky Way: Stellar Kinematics and the Microlensing Event Timescale Distribution in the Galactic Bulge. The Astrophysical Journal Letters, 601 . L155-L158. ISSN 2041-8205

It is advisable to refer to the publisher's version if you intend to cite from the work. http://dx.doi.org/10.1086/382043

For more information about UCLan's research in this area go to http://www.uclan.ac.uk/researchgroups/ and search for <name of research Group>.

For information about Research generally at UCLan please go to http://www.uclan.ac.uk/research/

All outputs in CLoK are protected by Intellectual Property Rights law, including Copyright law. Copyright, IPR and Moral Rights for the works on this site are retained by the individual authors and/or other copyright owners. Terms and conditions for use of this material are defined in the policies page.

\section{CLoK}

Central Lancashire online Knowledge www.clok.uclan.ac.uk

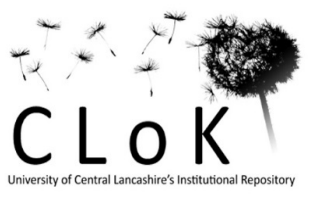


The Astrophysical Journal, 601:L155-L158, 2004 February 1

(C) 2004. The American Astronomical Society. All rights reserved. Printed in U.S.A.

\title{
LARGE-SCALE MODEL OF THE MILKY WAY: STELLAR KINEMATICS AND THE MICROLENSING EVENT TIMESCALE DISTRIBUTION IN THE GALACTIC BULGE
}

\author{
Nicolai Bissantz, ${ }^{1}$ Victor P. Debattista, ${ }^{2}$ and Ortwin Gerhard \\ Astronomisches Institut, Universität Basel, Venusstrasse 7, CH-4102 Binningen, Switzerland; gerhard@astro.unibas.ch \\ Received 2003 September 19; accepted 2003 December 15; published 2004 January 28
}

\begin{abstract}
We build a stellar-dynamical model of the Milky Way's barred bulge and disk, using a newly implemented adaptive particle method. The underlying mass model has been previously shown to match the Galactic nearinfrared surface brightness as well as gas-kinematic observations. Here we show that the new stellar-dynamical model also matches the observed stellar kinematics in several bulge fields and that its distribution of microlensing event timescales reproduces the observed timescale distribution of the MACHO experiment with a reasonable stellar mass function. The model is therefore an excellent basis for further studies of the Milky Way. We also predict the observational consequences of this mass function for parallax shifted events.
\end{abstract}

Subject headings: Galaxy: bulge — Galaxy: disk — Galaxy: kinematics and dynamics — Galaxy: structure

\section{INTRODUCTION}

It is now known, from several independent lines of evidence, that the Milky Way (MW) is barred (e.g., Gerhard 2001). However, a comprehensive model consistent with the main observables-luminosity distribution, stellar kinematics, gas kinematics, and microlensing - has so far been still missing. Recently, Bissantz \& Gerhard (2002) obtained a luminosity density model for the MW from the dust-corrected $L$-band COBE/DIRBE map of Spergel, Malhotra, \& Blitz (1996), through a nonparametric constrained maximum likelihood deprojection. This model (hereafter the COBE- $\rho$ model) is also consistent with the observed magnitude distributions of clump giant stars toward several bulge fields and with the microlensing optical depth toward the bulge derived from these stars (Popowski et al. 2004; Afonso et al. 2003); see also Binney, Bissantz, \& Gerhard (2000) and Bissantz \& Gerhard (2002). Furthermore, Bissantz, Englmaier, \& Gerhard (2003) found that the hydrodynamical gas flow in the potential of the COBE- $\rho$ model matches the observed gas dynamics of the inner MW well.

The structure of the inner MW can also be constrained by observations of stellar kinematics along fixed lines of sight (Sharples, Walker, \& Cropper 1990, hereafter SWC90; Spaenhauer, Jones, \& Whitford 1992, hereafter SJW92; Minniti et al. 1992, hereafter M92) and by the microlensing event timescale distribution (ETD; Alcock et al. 2000). The ETD has been studied largely with models that assume some distribution of disk and bulge kinematics (e.g., Han \& Gould 1996; Peale 1998; Méra, Chabrier, \& Schaeffer 1998). An exception was Zhao, Rich, \& Spergel (1996), who used the dynamical bar model of Zhao (1996) augmented by an analytic disk model but failed to match the long-duration $(\hat{t}>100$ days) tail of the ETD. In the present Letter, we show that a full stellar-dynamical model based on the $C O B E-\rho$ model is consistent with these independent data as well.

Dynamical models of the MW have been generated using the Schwarzschild (1979) method, in which the distribution function of a galaxy is built from numerically integrated stellar

\footnotetext{
Current address: Institut für Mathematische Stochastik, Maschmühlenweg 8-10, 37083 Göttingen, Germany; bissantz@math.uni-goettingen.de.

${ }^{2}$ Current address: Institut für Astronomie, ETH Hönggerberg, HPF G4.2, CH-8093 Zürich, Switzerland; debattis@phys.ethz.ch.
}

orbits. Following earlier work by Zhao (1996), Häfner et al. (2000) constructed a 22,168 orbit dynamical model of the MW. Dynamical models of the MW have also been obtained by $N$ body methods (Fux 1997). Syer \& Tremaine (1996, hereafter ST96) introduced a novel method for generating self-consistent dynamical models. The Syer-Tremaine (ST) method is allied to the Schwarzschild method, but rather than superposing timeaveraged observables from an orbit library, the ST method constructs a model by actively varying the weights of individual particles (orbits) as a function of time. This permits arbitrary geometry and a larger number of orbits to be used in the model building. Our dynamical model for the COBE- $\rho$ density in the MW is constructed with the ST method, demonstrating its usefulness for real galaxy modeling. This Letter compares the model's bulge kinematics and microlensing ETD with their observed counterparts.

\section{THE ST METHOD}

The idea of the ST algorithm is to assign individual weights to particles of a simulation, which are then changed to reduce the deviation between the model and observations. An observable $Y_{j}$ associated to a stellar system characterized by a distribution function $f(z), z=(\boldsymbol{x}, \boldsymbol{v})$ can be written as $Y_{j}=$ $\int K_{j}(z) f(z) d^{6} z$, where $K_{j}(z)$ is a known kernel. If this stellar system is simulated with $N$ particles having weights $w_{i}$ and phase-space coordinates $z_{i}$, then we can write the observables of the simulation as $y_{j}(t)=\sum_{i=1}^{N} w_{i}(t) K_{j}\left[z_{i}(t)\right]$. ST96 define the "force of change" on the weights as

$$
\frac{d w_{i}(t)}{d t}=-\epsilon w_{i}(t) \sum_{j} \frac{K_{j}\left(z_{i}\right)}{Z_{j}}\left[\frac{y_{j}(t)}{Y_{j}}-1\right]
$$

The small and positive parameter $\epsilon$ governs how rapidly the weights are pushed such that the simulation observables $y_{j}(t)$ converge toward the observables $Y_{j}$. The constants $Z_{j}$ act as normalizations. The full ST method also includes prescriptions for temporal smoothing and a maximum entropy term to reduce fluctuations. We have implemented the ST method with the MW disk-plane surface density as the observable (V. P. Debattista et al. 2004, in preparation). We set $\epsilon=0.25, \alpha=$ 0.524 , and $\mu=0.001$, where $\alpha$ and $\mu$ are the parameters of 
the temporal smoothing and the entropy terms, respectively, in the notation of ST96.

\subsection{Simulation}

Since the MW contains a bar, our initial model also had to be barred. The simplest way to achieve this was to evolve an $N$-body model of an initially axisymmetric bar-unstable disk galaxy. The $N$-body simulation that produced the barred model consisted of live disk and bulge components inside a frozen halo. The frozen halo was represented by a cored logarithmic potential. The initially axisymmetric disk was modeled by a truncated exponential disk. Disk kinematics were set up using the epicyclic approximation to give Toomre $Q=1.3$. The disk and bulge were represented by $4 \times 10^{6}$ equal-mass particles, with a mass ratio $M_{d}: M_{b}=4: 1$. Further details of the setup methods and model units can be found in Debattista (2003, hereafter D03). We use the halo, disk, and bulge parameters given in Table 2 of D03, which give a flat rotation curve out to large radii.

The simulation was run on a three-dimensional cylindrical polar grid code (described in Sellwood \& Valluri 1997), with technical parameters exactly as in D03. The initially axisymmetric system was unstable and formed a rapidly rotating bar at $t \simeq 50$. By $t=160$, the bar instability had run its course and further secular evolution of the bar was mild. The resulting system did not match the COBE- $\rho$ model of the MW and needed to be evolved further with the ST code. First, however, we eightfold symmetrized the $C O B E-\rho$ model in order to reduce the amplitude of spirals, which we did not try to reproduce. We evolved the $N$-body model from $t=160$ under the ST prescription with the fixed potential of the $C O B E-\rho$ model plus a dark matter halo. We kept the bar pattern speed at its value in the $N$-body model, which scales to $56 \mathrm{~km} \mathrm{~s}^{-1} \mathrm{kpc}^{-1}$, consistent with the MW (Dehnen 2000; Debattista, Gerhard, \& Sevenster 2002; Bissantz et al. 2003). At $t=240$ (i.e., $\simeq 4$ bar rotations), we shut off the ST algorithm and evolved the system to $t=280$ to assure that the particles are phase mixed.

\section{RESULTS: DENSITY AND BULGE KINEMATICS}

To compare our dynamical $C O B E$ model (the COBE-Dyn model) with observations, we adopted the same viewing parameters as were used to determine the $C O B E-\rho$ model: $R_{\odot}=8 \mathrm{kpc}, z_{\odot}=14 \mathrm{pc}$, and $\varphi_{\mathrm{bar}}=20^{\circ}$ (Bissantz \& Gerhard 2002). We scale the velocities in the $C O B E$-Dyn model to the MW by matching to the local circular velocity. We assumed that the local standard of rest has only a circular motion, with $v_{\text {LSR }}=200 \mathrm{~km} \mathrm{~s}^{-1}$, and we adopted the values of the solar peculiar motion from Dehnen \& Binney (1998).

The densities of the COBE-Dyn and COBE- $\rho$ models match very well, with azimuthally averaged errors smaller than $5 \%$ out to $R_{\odot}$. The largest errors $(<15 \%)$ occur in small isolated regions on the bar major axis. In the (unconstrained) vertical direction, the disk is somewhat thicker than the MW at $R_{\odot}$, but this leads to a change in optical depth $\tau$ toward Baade's window of less than $15 \%$. In the bulge region, on the other hand, the scale height of the COBE-Dyn model matches that of the MW very well. We compared the model's kinematics to observations toward Baade's window (SWC90; SJW92) and in the field at $(l, b)=$ $\left(8^{\circ}, 7^{\circ}\right)$ (M92), using the selection functions determined by Häfner et al. (2000). Table 1 shows our results. The overall fit of our model to the observed kinematics is rather good.
TABLE 1

Comparison of Kinematic Quantities Computed From the COBE-Dyn MODEL With OBSERVATIONS

\begin{tabular}{lcccc}
\hline \hline Kinematic Quantities & $(l, b)$ & Reference & Observed & COBE-Dyn \\
\hline$v_{\text {los }}^{h}\left(\mathrm{~km} \mathrm{~s}^{-1}\right) \ldots \ldots \ldots$ & $\left(1^{\circ},-4^{\circ}\right)$ & SWC90 & $4 \pm 8$ & 8.2 \\
$\sigma_{\text {los }}\left(\mathrm{km} \mathrm{s}^{-1}\right) \ldots \ldots \ldots$ & $\left(1^{\circ},-4^{\circ}\right)$ & SWC90 & $113 \pm 5$ & 109 \\
$\sigma_{\text {los }}\left(\mathrm{km} \mathrm{s}^{-1}\right) \ldots \ldots \ldots$ & $\left(1^{\circ},-4^{\circ}\right)$ & SJW92 & 120 & 109 \\
$\sigma_{\mu_{l}}^{h}\left(\mathrm{mas} \mathrm{yr}^{-1}\right) \ldots \ldots \ldots$ & $\left(1^{\circ},-4^{\circ}\right)$ & SJW92 & $3.2 \pm 0.1$ & 3.1 \\
$\sigma_{\mu_{b}}^{h}\left(\mathrm{mas} \mathrm{yr}^{-1}\right) \ldots \ldots \ldots$ & $\left(1^{\circ},-4^{\circ}\right)$ & SJW92 & $2.8 \pm 0.1$ & 2.4 \\
$v_{\text {los }}^{g}\left(\mathrm{~km} \mathrm{~s}^{-1}\right) \ldots \ldots \ldots$ & $\left(8^{\circ}, 7^{\circ}\right)$ & M92 & $45 \pm 10$ & 46 \\
$\sigma_{\text {los }}\left(\mathrm{km} \mathrm{s}^{-1}\right) \ldots \ldots \ldots$ & $\left(8^{\circ}, 7^{\circ}\right)$ & M92 & $85 \pm 7$ & 100 \\
\hline
\end{tabular}

Note. - In the first column, the superscript $h$ indicates that the value given is heliocentric, and $g$ that it is Galactocentric.

\section{THE MICROLENSING ETD}

We now show that the $C O B E$-Dyn model is also consistent with the microlensing ETD. Alcock et al. (2000) presented an ETD, corrected for their experimental detection efficiency, based on 99 events in eight fields. Popowski (2002) argued that one of these fields seems biased toward long-duration events, introducing some uncertainty in the observed ETD. Here we use the full-sample Alcock et al. ETD in order that our results may be compared with previous ones. We computed the ETD with the self-consistent kinematics of the COBE-Dyn model. A microlensing event is characterized by the source distance, $D_{S}$, lens distance, $D_{L}$, the proper motion, $v_{\perp}$, of the lens with respect to the line of sight between observer and source, and lens mass, $M_{L}$. The probability $P(\hat{t})$ for observing an event duration $\hat{t}=2 \Theta_{\mathrm{E}} / v_{\perp}$ is given by

$$
\begin{aligned}
P(\hat{t}) \propto & \int \rho\left(D_{S}\right) D_{S}^{2+2 \beta} \rho\left(D_{L}\right) D_{L}^{2} \Theta_{\mathrm{E}}\left(D_{S}, D_{L}, M_{L}\right) \\
\times & \Phi\left(M_{L}\right) v_{\perp} f\left(v_{\perp}\right) \delta\left(\hat{t}-2 \Theta_{\mathrm{E}} / v_{\perp}\right) d v_{\perp} d D_{L} d D_{S} d M_{L}
\end{aligned}
$$

Here $\rho(d)$ is the density of the MW at distance $d$ from the observer along the line of sight to the observed field, $\Phi\left(M_{L}\right)$ is the mass function (MF) of the lens population, $\Theta_{\mathrm{E}}\left(D_{S}\right.$, $\left.D_{L}, M_{L}\right)$ is the Einstein angle, and $f\left(v_{\perp}\right)$ is the distribution of $v_{\perp}$. We solved the multiple integral by Monte Carlo random drawings of the parameters $\left(D_{S}, D_{L}, v_{\perp}, M_{L}\right)$ as follows. (1) To obtain the source distance $\left(0 \leq D_{S} \leq D_{S}^{\max }=12 \mathrm{kpc}\right)$, we used the $C O B E-\rho$ model, since this is less noisy than the particle realization. The probability of $D_{S}$ is proportional to $\rho\left(D_{S}\right) D_{S}^{2+2 \beta}$ with $\beta=-1$, to account for a magnitude cutoff (Kiraga \& Paczyński 1994). (2) The lens distance $\left(0 \leq D_{L}<\right.$ $\left.D_{S}\right)$ was selected from $\tilde{\rho}\left(D_{L}\right) \int_{0}^{D_{S}} \rho\left(D_{L}\right) d D_{L}$, where $\tilde{\rho}$ is a normalized probability density distributed as in the COBE- $\rho$ model. (3) For the relative velocity $v_{\perp}$, we used the particle distribution of the COBE-Dyn model, randomly selecting a particle at $\sim D_{S}$ and another at $\sim D_{L}$. The proper motions of these particles then determined $v_{\perp}$. (4) The lens mass $M_{L} / M_{\odot}$ was selected from a Kroupa (1995) MF, $\Phi\left(M_{L}\right)=\Phi_{0}\left(M_{L} / M_{\odot}\right)^{-\gamma}$, with

$$
\left(\gamma, \Phi_{0}\right)= \begin{cases}(2.35,0.1038), & M_{L}^{<} \leq M_{L} / M_{\odot} \leq 0.35 \\ (0.6,0.6529), & 0.35 \leq M_{L} / M_{\odot} \leq 0.6 \\ (2.35,0.2674), & 0.6 \leq M_{L} / M_{\odot} \leq M_{L}^{>}\end{cases}
$$

We explored varying $M_{L}^{<}$and $M_{L}^{>}$. We obtained the ETD, shown in Figure 1, by simulating $10^{5}$ events and weighting each by the remaining factors in equation (2). We tested our Monte 


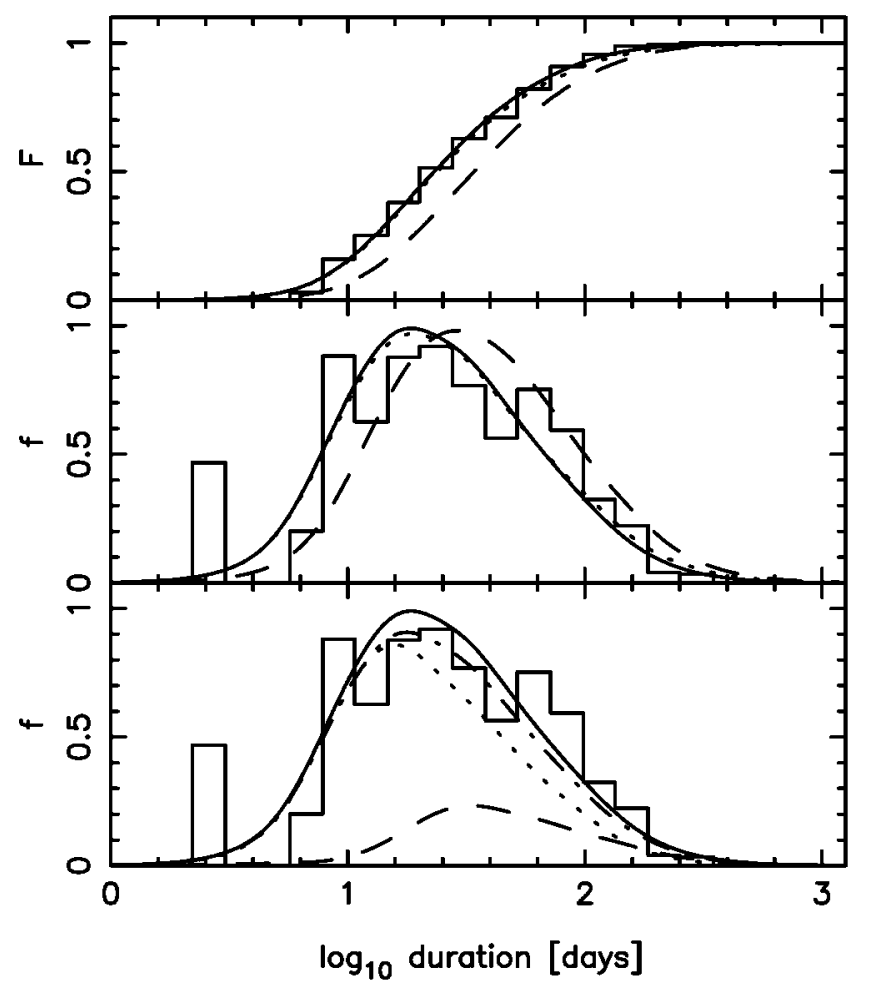

FIG. 1.-ETD of the COBE-Dyn model compared to the detectionefficiency-corrected observations of the MACHO group (histograms in all panels). Top: Cumulative distribution function for the standard model, $\left(M_{L}^{<}, M_{L}^{\odot}\right)=(0.04,4)($ solid line $)$, the best model with $\left(M_{L}^{<}, M_{L}^{\odot}\right)=(0.04,10)$ (dotted line), and a model with $\left(M_{L}^{<}, M_{\perp}\right)=(0.075,10)$ (dashed line). We obtain $D_{\mathrm{KS}}=0.081,0.068,0.213$, respectively, for the three models. Middle: Differential distributions of these models (same line styles). (In these two panels, all model distributions have been smoothed with a kernel density estimator of bandwidth 0.1.) Bottom: ETD of the $\left(M_{L}^{<}, M_{L}^{\triangleright}\right)=(0.04,4)$ model and its decomposition into events with $6 \leq D_{S} \leq 10 \mathrm{kpc}$ (dotted line), $0 \leq$ $D_{L} \leq 4 \mathrm{kpc}$ (dashed line), and $D_{L}>4 \mathrm{kpc}$ (dot-dashed line).

Carlo integrations by reproducing one of the model ETDs in Peale (1998).

We started with $\left(M_{L}^{<}, M_{L}^{>}\right)=(0.075,10)$, for which we obtained a Kolmogorov-Smirnov distance between data and model of $D_{\mathrm{KS}}=0.213$. (We excluded the bin at $\hat{t}<3.1$ days from the MACHO data in all such comparisons, because it appears to be too heavily affected by its large detectionefficiency correction.) To improve on this fit, we first explored the effects of uncertainties in the $C O B E-\rho$ model. The most important of these is $\varphi_{\text {bar }}$. Setting $\varphi_{\text {bar }}=30^{\circ}$, we found only a minor change to the ETD, in agreement with Peale (1998). Making the bar stronger or the disk velocity dispersion outside the bar smaller did not alter the ETD substantially. Therefore we next explored variations in the MF. Like Peale (1998), we found that modest changes can improve the fit substantially. Our best fit with $D_{\mathrm{KS}}=0.068$ was obtained with $M_{L}^{<}=0.04$ and $M_{L}^{>}=10$. However, a more conservative limit is $M_{L}^{>}=$ 4, which gives $D_{\mathrm{KS}}=0.081$. (If the suggestion of Popowski 2002 is correct, which would shift the ETD peak to smaller $\hat{t}$, then a smaller $M_{L}^{>}$would be required anyway.)

We now explore the causes of long-duration (LD) events in the COBE-Dyn model, using $\left(M_{L}^{<}, M_{L}^{>}\right)=(0.04,4)$ as our standard model for this analysis. We start by noting, from Figure 1 , that the vast majority of sources are located in the bulge $\left(6 \mathrm{kpc} \leq D_{S} \leq 10 \mathrm{kpc}\right)$. This is also true for the lenses respon-
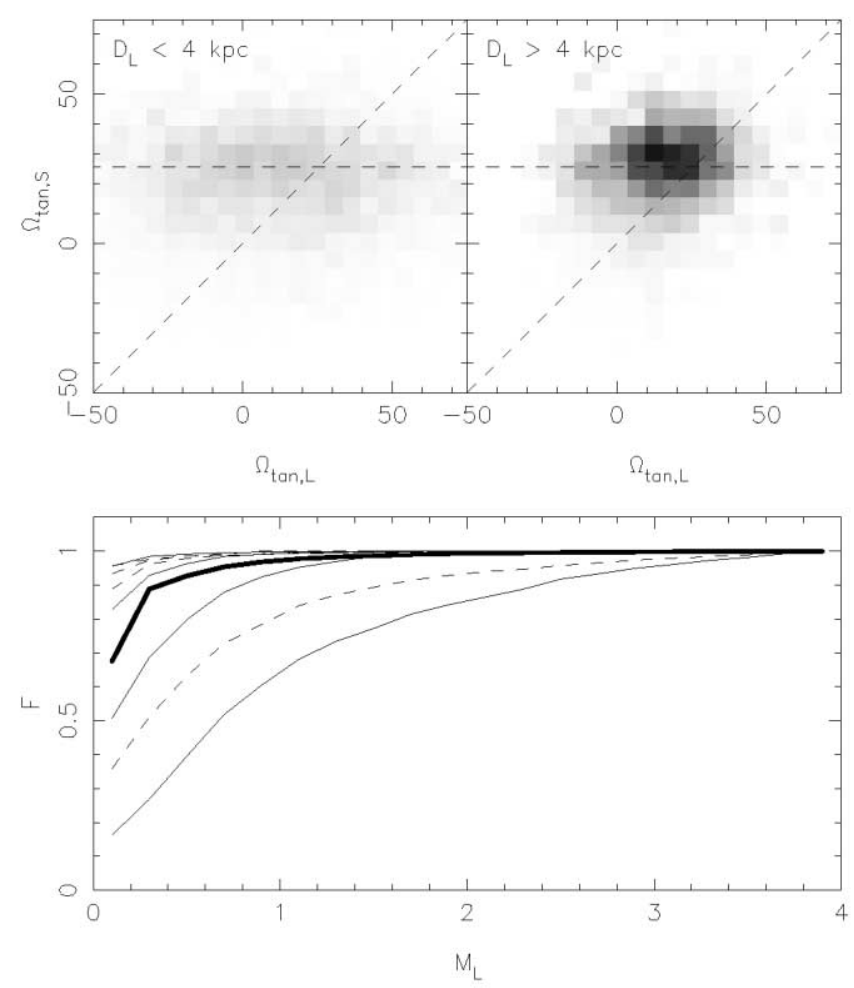

Fig. 2.-Top two panels: LD events $(\hat{t}>42$ days $)$ in the $\left(M_{L}^{<}, M_{L}^{>}\right)=$ $(0.04,4)$ model. Both are maps (on the same relative scale) in the plane of heliocentric tangential angular velocities, $\Omega_{\tan , L}$ and $\Omega_{\tan , s}$. Left: Near lenses $\left(D_{L}<4 \mathrm{kpc}\right)$; right: distant ones $\left(D_{L}>4 \mathrm{kpc}\right)$. The diagonal and horizontal dashed lines indicate $\Omega_{\tan , L}=\Omega_{\tan , S}$ and $\Omega_{\tan , S}=205 / 8=25.6 \mathrm{~km} \mathrm{~s}^{-1} \mathrm{kpc}^{-1}$, respectively. Bottom: Distributions of $M_{L}$ for distant (solid lines) and nearby (dashed lines) lenses. The different lines result from splitting into quartiles by contribution to the full ETD the distribution of events sorted on $\hat{t}$. Event durations increase as the mean mass increases. The heavy curve shows the underlying mass function.

sible for short duration events, but disk lenses become more important at longer durations; indeed, for $\hat{t}>25$ days, one-third of the lenses are at $D_{L}<4 \mathrm{kpc}$. In Figure 2 we separate the ETD into the near and distant lens subsamples and show the heliocentric angular velocities and cumulative distributions of $M_{L}$ for both. Note first that lenses with $M_{L}>0.5 M_{\odot}$ contribute significantly to LD events in both the near and the distant subsamples. Lens mass, however, is not the full explanation of the LD events, as has been noted by previous studies, and the relative motions of lens and source in the heliocentric frame must also be considered. The kinematics of the LD sources are substantially those of a rotating triaxial bulge/bar that points almost toward the observer: thus their apparent tangential motions are due largely to the solar motion, giving $\Omega_{\tan , S} \sim$ 205/8 $\mathrm{km} \mathrm{s}^{-1} \mathrm{kpc}^{-1}$. Distant lens LD events are then possible because the lenses share very similar kinematics with the sources (note that massive lenses become necessary only in the last quartile, $\hat{t}>60$ days). For the nearby lens sample, LD events have a rather large spread in $\Omega_{\tan , L}$ (due to both their proximity and the velocity dispersion of the COBE-Dyn model), which together with larger $M_{L}$ is able to produce $\mathrm{LD}$ events. We conclude, therefore, that there is no single cause for the LD events.

Standard three-parameter fits to microlensing events are symmetric about the time of peak amplification, resulting in a degeneracy among $M_{L}, v_{\perp}, D_{L}$, and $D_{S}$. One degree of degeneracy 
is removed by also measuring the light-curve shift due to the parallax from earth's orbit, which gives a relation between $v_{\perp}$ and $D_{L} / D_{S}$. These shifts are present in all events, but most go undetected because of infrequent sampling and photometric errors. Buchalter \& Kamionkowski (1997) estimate a $1 \%$ detection efficiency of parallax-shifted events for the MACHOtype setup and much higher for second-generation experiments. The light curves of such events require five parameters, including $\kappa \equiv R_{\oplus}\left(D_{L}^{-1}-D_{S}^{-1}\right) / \Theta_{\mathrm{E}}$, where $R_{\oplus}=1$ AU. In Figure 3, we present our predictions for the probability distribution in the $(\kappa, \hat{t})$-plane, assuming $100 \%$ detection efficiency. These distributions are twin-peaked, with the lower peak increasingly separated from the global peak as $M_{L}^{<}$decreases, as it must since $\kappa \propto \theta_{\mathrm{E}}^{-1}$ while $\hat{t} \propto \Theta_{\mathrm{E}}$. The location of the second peak may therefore provide an observational constraint on the MF at low mass.

\section{CONCLUSIONS}

We have presented a dynamical model of the MW constructed using the Syer-Tremaine method, constrained only by the MW density map of Bissantz \& Gerhard (2002). Although no kinematic constraints were used, the model (1) matches observed bulge kinematics in several fields and is (2) able to reproduce the observed microlensing EDT. For the best-fitting MF, the model (3) predicts a twin-peaked probability distribution in the $(\kappa, \hat{t})$-plane, which may be observationally tested with new generations of microlensing experiments. (4) The underlying mass model has been previously shown to match the Galactic near-infrared surface brightness as well as gas-

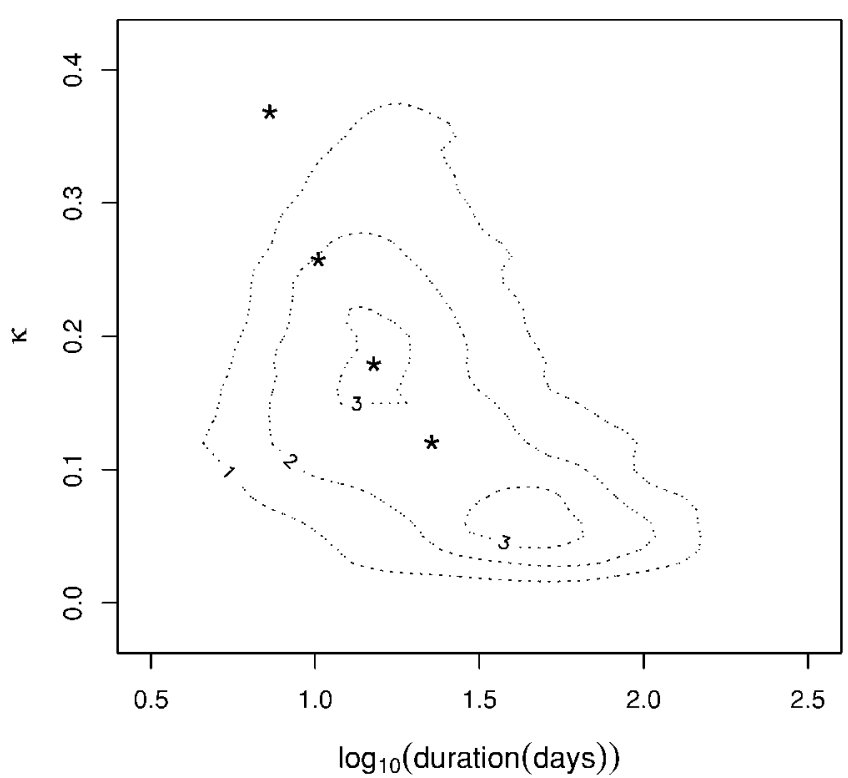

FIG. 3.-Predicted probability distribution of parallax-shifted events in the $(\kappa, \hat{t})$-plane, for the standard model with $\left(M_{L}^{<}, M_{L}^{>}\right)=(0.04,4)$. We use a smoothing kernel with $\left(\delta_{\kappa}, \delta_{\log \hat{t}}\right)=(0.01,0.1)$. The asterisks mark the locations of secondary peaks when $M_{L}^{<}=0.075,0.04,0.02$, and 0.01 in order of increasing $\kappa$.

kinematic observations. It is therefore an excellent basis for further studies of the Milky Way.

This work was supported by the Schweizerischer Nationalfonds through grant 20-64856.01.

\section{REFERENCES}

Afonso, C., et al. 2003, A\&A, 404, 145

Alcock, C., et al. 2000, ApJ, 541, 734

Binney, J. J., Bissantz, N., \& Gerhard, O. E. 2000, ApJ, 537, L99

Bissantz, N., Englmaier, P., \& Gerhard, O. E. 2003, MNRAS, 340, 949

Bissantz, N., \& Gerhard, O. E. 2002, MNRAS, 330, 591

Buchalter, A., \& Kamionkowski, M. 1997, ApJ, 482, 782

Debattista, V. P. 2003, MNRAS, 342, 1194 (D03)

Debattista, V. P., Gerhard, O., \& Sevenster, M. N. 2002, MNRAS, 334, 355

Dehnen, W. 2000, AJ, 119, 800

Dehnen, W., \& Binney, J. 1998, MNRAS, 298, 387

Fux, R. 1997, A\&A, 327, 983

Gerhard O. 2001, in ASP Conf. Ser. 230, Galaxy Disks and Disk Galaxies, ed. J. G. Funes, S.J., \& E. M. Corsini (San Francisco: ASP), 21

Häfner, R., Evans, N. W., Dehnen, W., \& Binney, J. 2000, MNRAS, 314, 433

Han, C., \& Gould, A. 1996, ApJ, 473, 230

Kiraga, M., \& Paczyński, B. 1994, ApJ, 430, L101

Kroupa, P. 1995, ApJ, 453, 358
Méra, D., Chabrier, G., \& Schaeffer, R. 1998, A\&A, 330, 937

Minniti, D., White, S. D. M., Olszewski, E. W., \& Hill, J. M. 1992, ApJ, 393, L47 (M92)

Peale, S. J. 1998, ApJ, 509, 177

Popowski, P. 2002, MNRAS, submitted (astro-ph/0205044)

Popowski, P., et al. 2004, in Gravitational Lensing: A Unique Tool for Cosmology, ed. D. Valls-Gabaud \& J.-P. Kneib, in press (astro-ph/0304464)

Schwarzschild, M. 1979, ApJ, 232, 236

Sellwood, J. A., \& Valluri, M. 1997, MNRAS, 287, 124

Sharples, R., Walker, A., \& Cropper, M. 1990, MNRAS, 246, 54 (SWC90)

Spaenhauer, A., Jones, B. F., \& Whitford, A. E. 1992, AJ, 103, 297 (SJW92)

Spergel, D. N., Malhotra, S., \& Blitz, L. 1996, in Spiral Galaxies in the Near-

IR, ed. D. Minniti \& H.-W. Rix (Berlin: Springer), 128

Syer, D., \& Tremaine, S. 1996, MNRAS, 282, 223

Zhao, H.-S. 1996, MNRAS, 283, 149

Zhao, H.-S., Rich, R. M., \& Spergel, D. N. 1996, MNRAS, 282, 175 